\title{
Robotic and artificial intelligence for keyhole neurosurgery: the ROBOCAST project, a multi-modal autonomous path planner
}

\author{
E De Momi and G Ferrigno* \\ Bioengineering Department, Politecnico di Milano, Milano, Italy
}

The manuscript was received on 27 January 2009 and was accepted after revision for publication on 1 October 2009.

DOI: 10.1243/09544119JEIM585

\begin{abstract}
The robot and sensors integration for computer-assisted surgery and therapy (ROBOCAST) project (FP7-ICT-2007-215190) is co-funded by the European Union within the Seventh Framework Programme in the field of information and communication technologies. The ROBOCAST project focuses on robot- and artificial-intelligence-assisted keyhole neurosurgery (tumour biopsy and local drug delivery along straight or turning paths). The goal of this project is to assist surgeons with a robotic system controlled by an intelligent highlevel controller (HLC) able to gather and integrate information from the surgeon, from diagnostic images, and from an array of on-field sensors. The HLC integrates pre-operative and intra-operative diagnostics data and measurements, intelligence augmentation, multiple-robot dexterity, and multiple sensory inputs in a closed-loop cooperating scheme including a smart interface for improved haptic immersion and integration. This paper, after the overall architecture description, focuses on the intelligent trajectory planner based on risk estimation and human criticism. The current status of development is reported, and first tests on the planner are shown by using a real image stack and risk descriptor phantom. The advantages of using a fuzzy risk description are given by the possibility of upgrading the knowledge on-field without the intervention of a knowledge engineer.
\end{abstract}

Keywords: robotic assisted surgery, artificial intelligence, keyhole neurosurgery, ROBOCAST project

\section{INTRODUCTION}

The robot and sensors integration for computerassisted surgery and therapy (ROBOCAST) project (FP7-ICT-2007-215190) is a Specific Targeted Research Project co-funded by the European Union (EU) within the Seventh Framework Programme (FP7) in the field of information and communication technologies (ICT). Within this programme, Challenge 2, 'Cognitive systems, interaction, robotics', aims at creating the next generation of ICT intelligent systems and products, with the aim of opening the way to a wide range of opportunities for ICT-

*Corresponding author: NearLab, Bioengineering Department, Politecnico di Milano, Piazza Leonardo da Vinci 32, Milano 20133, Italy.

email: giancarlo.ferrigno@polimi.it based applications in a wider range of sectors, including healthcare, as in the case of the ROBOCAST project.

Industrial robots have reached maturity in terms of efficacy, efficiency, and cost. Today's challenges in robotics stem from the need to exploit robotic technology in risky, safety-critical, and demanding sectors, such as space exploration and interventional medicine. The latter is particularly demanding in terms of safety, accuracy, miniaturization, working space, low encumbrance, and interaction requirements for performing critical and tiring tasks in hazardous situations and uncertain environments. Surgery is thus a perfect test bench for new ICT approaches which can be extended to very different application fields. Analysing the rationale behind the slow uptake of systems in surgical robotics in general, and neurosurgical robotics in particular, is 
relevant to the project. Several reasons may be responsible for the demise of otherwise impressive systems [1-4]. Principally, these are:

(a) safety concerns with regard to both the patient and the user, due to the lack of intra-operative information or imaging and to error interpretation and visualization;

(b) an excessive footprint (obtrusiveness);

(c) the predominantly passive nature of their function within the operating theatre;

(d) the limitations of instruments (e.g. straight-line paths of conventional probes);

(e) poor integration with other instruments in the operating room (OR) and in the routine surgical workflow;

(f) poor cost-effectiveness of equipment and if systems are not usable for other interventions additional intra-operative time required for the robot-assisted procedure.

Recently, Pandya et al. [5] tested the NeuroArm, which is an image-guided magnetic-resonance (MR)-compatible surgical robot capable of both microsurgery and stereotaxy, and tested with clinical studies. Even if first results are promising, MRcompatible systems are highly costly. Xia et al. [6] are integrating a commercially available robotic system (NeuroMate) with optical tracking and surgeon cooperative control. A real-time application interface makes it possible to avoid encumbering patient head fixation but feedback control time delay results are not reported.

\subsection{The ROBOCAST scenario}

The advantages of minimally invasive surgery (MIS) for the patient are unquestionable. As the opening is smaller, MIS causes less pain and stress to the organism. In turn, this results in faster recovery, lower risk of infection, less scarring, and the economic gains that result from a shorter illness time. However, when performed by conventional means, MIS allows only restricted vision in the operating theatre, difficult and tiring handling of the instruments, restricted mobility, difficult hand-eye coordination with little or no tactile perception, and reduced accuracy and repeatability during the handling of the MIS instrument set. As a result, MIS procedures are complex and error prone; therefore a conventional wide-open intervention is often favoured to reduce the risk of complications, the required time, and the stressing concentration.
The ROBOCAST project focuses on robot-assisted keyhole neurosurgery. This latter term refers to brain surgeries performed through a very small hole in the skull (approximately $2 \mathrm{~cm}$ in diameter) called a burr hole or 'keyhole' [7]. Needles and catheters are inserted into the brain for biopsy and therapy, including, among others, the tasks of blood or fluid sampling, cryogenic and electrolytic ablation, brachytherapy, deep brain stimulation (DBS), diagnostic imaging, and a number of other MIS procedures (Fig. 1). Related pathologies are tumours, hydrocephalus, dystonia, essential tremor, Parkinson's disease, Tourette's syndrome, clinical depression, phantom limb pain, cluster headache, and epilepsy. The ROBOCAST project aims at advancing the current state of the art in neurosurgical technology by addressing the above-cited shortcomings of current surgical devices.

Two paradigmatic scenarios were defined by the consortium as a test bench for the ROBOCAST system. Since the project foresees a straight trajectory planner and a proof of concept of a flexible probe, the first scenario is based on a straight trajectory with possible stop points along the way, while the second is based on curvilinear trajectories. The first scenario is typical of (possibly multiple) tumour biopsy and local drug delivery, while the second can be either a biopsy characterized by nonlinear trajectories or a highly toxic chemotherapy treatment that can be combined with other methods (e.g. radioactive seeds, cryotherapy, or hyperthermia) on multiple targets of the same lesion for the optimization of the drug delivery, avoiding the scattering of chemicals along the liquoral ducts. It also allows brain liquor sampling before and after drug administration, by microdialysis, in order to

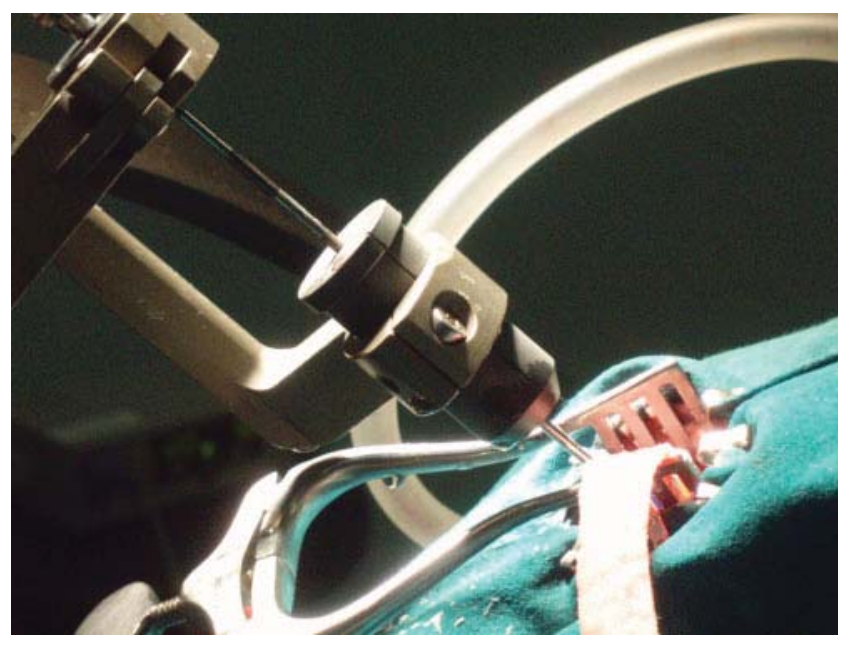

Fig. 1 Current operative scenario for keyhole biopsy 
investigate the drug feedback mechanism for treatment customization.

Because of the complexity of the morphology and of the functionality of the human brain, the planning of the best probe path, which causes less damage to brain structures, is a difficult task even for the experienced surgeon. The abundance of imaging information increases the possibility of planning the trajectories better, but the overall data are difficult to tackle at once without intelligent support for the surgeon. Automatic planning software has recently been proposed, on the basis of a weighted costs function related to anatomical atlases. Vaillant et al. [8] proposed a method for finding the path that avoids, as much as possible, some critical regions defined using the Talairach brain atlas. Given a target in the brain, the entry point on the skull is computed so that the cost function, which non-linearly weights the importance of the tissue located along the trajectory (and therefore the risk incurred in damaging it), is minimized. Kaur et al. [9] assigned a priority value to 137 structures of the same atlas. The query was performed by using a variable-depth target tree that recursively decomposes the search space around a single target point. The aforementioned studies did not examine any statistical uncertainty in the considered atlas, and the information provided to the surgeon was limited to the brain tissue classification. A slightly different approach was followed by Brunenberg et al. [10], namely DBS applications. Important structures (blood vessels, ventricles, and gyri) were segmented on $\mathrm{T} 1$ and $\mathrm{T} 2 \mathrm{MR}$ images and computed tomography (CT) data sets. They constrained the entry region to the frontal lobe gyri, and then all the safe paths to the targets were considered, together with their distances from vessels and from ventricles. With this approach, the analysis was limited to visible and automatically detected structures. Also, functional information (derived from functional magnetic resonance imaging (fMRI)) and anatomical information (derived from diffusion tensor imaging (DTI)) had not yet been considered. The International Consortium for Brain Mapping (ICBM) has been working on the development of a probabilistic brain atlas [11], collecting images from 7000 subjects. Tissue (white matter, grey matter, and cerebrospinal fluid), lobular, deep nuclei, and sulcal probabilistic atlases contain the probability that a particular structure is present at a given location.

In this framework, the ROBOCAST system provides a tool for automatic planning of the best probe path inside the brain, based on the minimization of the patient risk through multi-modal information sets and based on surgeon-dependent rules, stored in a knowledge repository.

\subsection{The ROBOCAST objectives}

The goal of the project is to assist surgeons with a robotic system controlled by an intelligent high-level controller (HLC) able to gather and integrate information from the surgeon, from diagnostic data, and from an array of on-field sensors. The HLC integrates pre-operative and intra-operative diagnostics data and measurements, intelligent augmentation (IA), multiple-robot dexterity, and multiple sensory inputs in a closed-loop cooperating scheme including a smart interface for improved haptic immersion and integration. The idea is being developed into a demonstrator for in-vitro experimentation (by the end of 2010). The specific scientific and technological objectives of the project are the following:

(a) to develop IA techniques for task performance and effective human-machine interaction (path planning and negotiation, uncertainty management, and intelligent interface);

(b) to provide robots with autonomy (management of failures, negotiation of path execution, and optical sensor management);

(c) to increase the basic accuracy and reliability of the robot by a feedback control loop and sensor-robot-robot cooperation;

(d) to develop an effective interface between the user and the system (user-robot ergonomic interface) and to provide a strong integration in the OR;

(e) to provide the system with modularity and flexibility characteristics to make it scalable and usable in different applications (e.g. surgical and industrial).

Objective (a) is oriented to overcome two problems that have prevented the wide diffusion of surgical robotics and that are common with other robotic approaches: first, the autonomous path planning and, second, the effective interfacing of an intelligent artificial system to non-skilled users. Path planning is indeed a key point in surgery as well as in autonomous environment exploration. Intelligent path planning relies on more information than the information that an end user may manage contemporaneously and thus may be faster and more reliable; furthermore it may spare surgical time, thus facilitating the acceptance of the overall system. Mandatorily, surgical planning requires a negotiation phase with the person uniquely responsible for the intervention, i.e. the surgeon. This opens an issue in interfacing an 
artificial intelligence system with a generally nonskilled user. In the ROBOCAST project, context-driven multiple-choice statements are used to allow such a negotiation and decisions explanation.

Objective (b) is directed towards the autonomy of planning and the autonomous management of failures. Because of the lack of reliability in the information from sensors, facing unexpected events is desirable for the surgical application and for other robotics fields where autonomous decisions must be taken. The surgeon is the last referee of the system actions, while in other, less crucial, applications the robot itself can take decisions, based on the current reliability of position information.

As objective (c), a wide use of redundant sensors is explored both for increasing system reliability and for compensating mechanical hardware inaccuracies, through a supplementary sensorial feedback.

Objective (d) is the integration of the system in existing instrumentation, a factor which plays a paramount role in the OR application, but it is also very suitable whenever a device is to be included in, and must cooperate with, something already existing.

The final objective (e) is the modularity, in terms of hardware and software, in order to allow reusing of any module of the project for other applications, and to facilitate maintenance and repair in the highly demanding surgical application.

\section{THE ROBOCAST DEMONSTRATOR}

\subsection{System architecture}

The ROBOCAST demonstrator integrates robot hardware, sensors, system controller, an autonomous planner, and a user interface (i.e. a human-computer interface (HCI)).

The approach herein followed is to sketch the overall system architecture and to deepen the analysis of the autonomous planner, which is one of the more challenging parts of the system and can have some fallouts in other robotic applications requiring optimal path determination.

Figure 2 shows the main components of the ROBOCAST demonstrator. The HCI allows the user (surgeon, physicist, or radiologist) to interact with the medical images in order to identify the target and to plan the surgical intervention. The ROBOCAST system in this framework helps with autonomous trajectory definition. Improved robotic architecture, including a flexible probe proof of concept, guarantees the flexibility, accuracy, and redundancy to make the system reliable and adaptable to the OR configuration. Independent sensors allow the relia- bility of the system to be increased and the controller to be provided with information about the robot positions and also about the force exerted on brain parenchyma.

\subsection{Robot hardware}

The positioning system of the ROBOCAST demonstrator includes three sections: gross positioner (GP), fine positioner (FP), and probe (linear or flexible). The rationale behind this choice is to split the control problem into delimited subproblems. The GP locates the FP in the proximity of the patient's body, positioning it in order to centre its full range of motion around the planned set point. This feature ensures that the FP does not reach the full range of motion during the operation. The FP is thus used to correct for the GP inaccuracies in positioning, allowing small GP robots to be used with possible inaccuracy on end effector positioning. A linear actuator (LA) or the flexible probe (FLP) trocar are positioned on top of the FP. The LA is equipped with a force sensor, while the FLP carries a magnetic sensor on the tip. A series of dynamic reference frames (DRFs), based on active markers, are used to locate the actuation hardware independently from the internal encoders, so that an increased reliability is obtained as well as more information for the controller algorithm.

\subsubsection{GP}

The GP used for the ROBOCAST demonstrator is the PathFinder [12]. This robot is designed for medical applications (laparoscopy and neurosurgery), and so it already has some characteristics suitable for the application, such as provision for sterility, via draping, quick interlocks for instruments with identification pins, and watchdogs. Indeed it is a very stiff and accurate device, while ROBOCAST foresees the possibility of using low-cost small-GP systems. This characteristic is simulated by adding random errors on the PathFinder end effector.

\subsubsection{FP}

A six-degrees-of-freedom parallel robot, MARS (Mazor Surgical Technologies Ltd, Cesarea, Israel) [13], is linked to the end effector of the GP. The task of this hexapod is to position the LA or the FLP trocar finely. Its accuracy and the redundant sensor feedback allow compensation of any errors of the GP.

\subsubsection{LA}

The LA advances the straight biopsy probe within the patient's brain along the planned trajectory. Because 


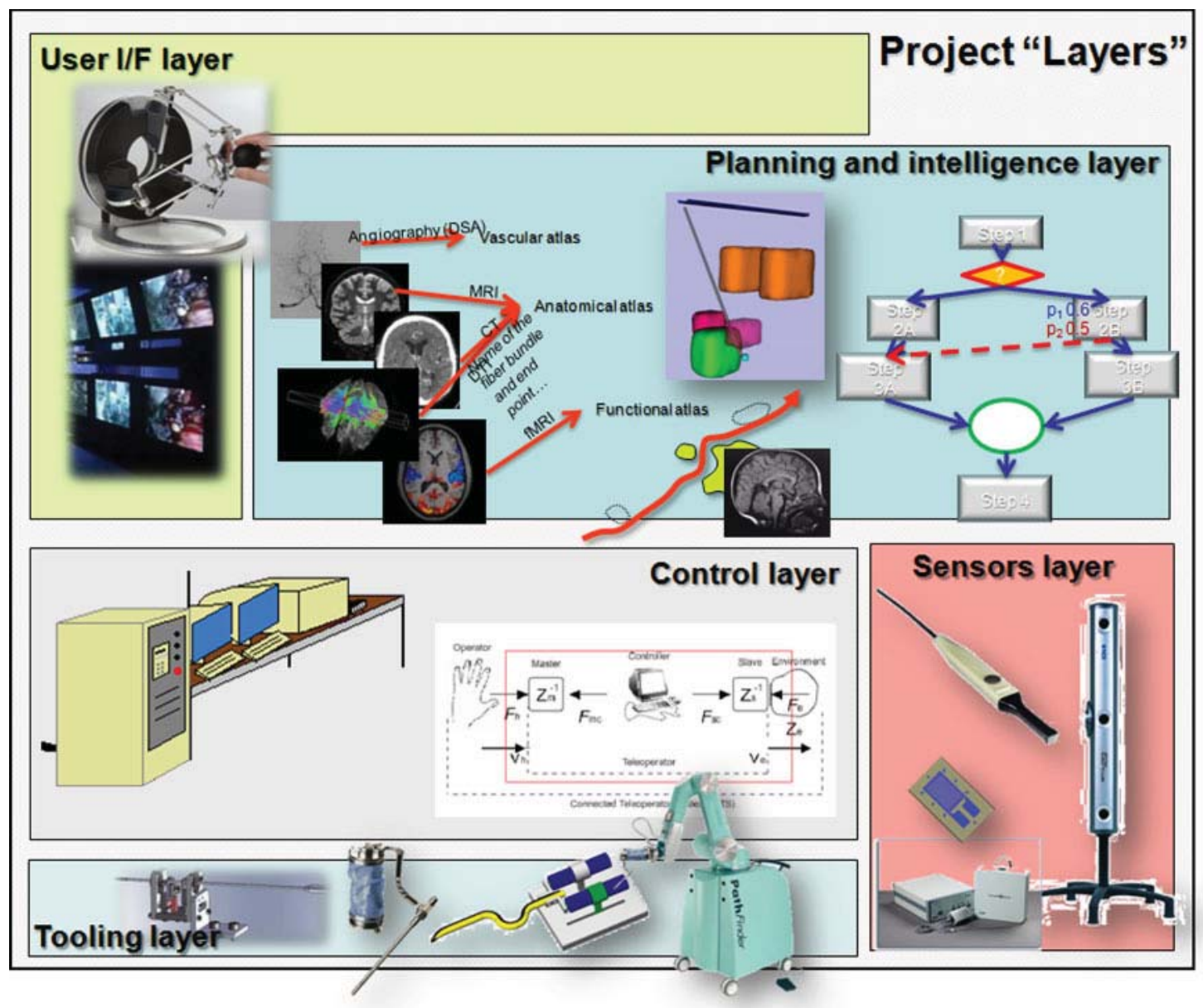

Fig. 2 The ROBOCAST architecture: user interface (HCI), planning and intelligence, system controller (HLC), sensors, and robot hardware

of sterilization requirements, a piezoelectric motor was utilized. The piezo motor allows both straight motion and a pulsating forward-backward micromotion in order to reduce friction with brain parenchyma. The LA is equipped with a redundant sensor system including a linear encoder and an optical DRF.

\subsubsection{FLP}

The FLP represents a high-risk development in the ROBOCAST project. It is based on a biomimetic solution [14] to surgical probe buckling (buckling is an instability phenomenon of a pipe when, owing to thermal or mechanical loads and inability to expand axially, it moves laterally, assuming a curved shape). A flexed probe pushed in a low-resistance medium, such as the brain, does not follow the curvature but tends to displace the medium itself, seriously damaging it. The biomimetic approach herein proposed uses a probe split into reciprocating parts that actively contribute to the advancement, and require a minimal inward push, thereby avoiding buckling. The probe consists of two halves forming one shaft; therefore it can act as a needle with two different bevels of the same angle on different sides. In doing this, a different steering system to trace curved trajectories is obtained. The mathematical modelling of this probe is achived as a differentialwheel-drive car with two active wheels. A minimum of three reciprocating parts would be required for a three-dimensional (3D) trajectory. In the ROBOCAST project, a two-dimensional (2D) demonstrator (i.e. a curved path in a plane) is foreseen. This is the more risky part of all the research and it is likely that a final, working, real-size device cannot be built. However, a proof of concept will be produced and tested. The trajectory of the tip of the FLP will be tracked inside the brain by a magnetic tracker.

\subsection{Sensor manager}

The sensor manager (SM) integrates the information coming from a group of field sensors: optical tracker, 
magnetic tracker, robot encoders, force sensor, intraoperative ultrasound (US) probe, and intra-operative Doppler US probe. The SM provides data collection and registration into the medical images reference frame, after calibration. Two localization systems are used and integrated: an optical system, Certus (NDI, Waterloo, Ontario, Canada), and an electromagnetic (EM) system, Aurora (NDI, Waterloo, Ontario, Canada). The optical system tracks the position and the orientation of all the rigid body with a clear line of sight towards the position sensor, while the EM system is specifically used only to track the tip of the FLP, once it is inserted in the brain tissue.

The optical tracker is a high-accuracy active marker optical localization system, with a stated accuracy of $0.1 \mathrm{~mm}$ in the 3D marker's location. The DRFs are fixed to the following:
(a) GP base;
(b) FP base;
(c) rigid probe handle;
(d) FLP trocar.

The magnetic tracker has a stated accuracy of $0.6 \mathrm{~mm}$ in position and 0.4 degrees for a six-degreesof-freedom coil system (within the working volume of $0.5 \mathrm{~m} \times 0.5 \mathrm{~m} \times 0.5 \mathrm{~m})$.

The force sensor will be mounted on the LA (strain gauges are mounted on the actuating jaws of the piezoelectric advancement mechanism) in order to check the resistance offered by the brain to the linear probe advancement. This information will be provided as haptic feedback to the surgeon's hand, and to check smooth and safe advancement by comparing the actual force with the expected force. Expected force information is derived from the literature and updated during use of the system by measuring actual forces in operations that went well. If the measured actual force is extremely different from the expected value, the probe advancement is automatically stopped. Force data constitute a further layer of information of the system.

Two US sensors are used. It is well known that opening meninges (dura mater) causes a spill of liquid that lets the brain collapse on the brain stem. This phenomenon is known as 'brain shift' and can invalidate a pre-operative plan for the target displacement. The use of registration of pre-operative and intra-operative images allows replanning with intra-operative data. Precise location of the US imaging plane is obtained from a DRF mounted on the US probe. The US probes are fed through the burr hole or through the temporal bone if it is sufficiently thin.
The second US probe is a miniaturized Doppler device (diameter, less than $2 \mathrm{~mm}$ ) used to detect small vessels during probe advancement. Vessel damage is, in fact, the major cause of morbidity in keyhole neurosurgery [15].

In order to manage all the sensors and to assure synchronization between all the gathered information, the Image-Guided Surgery Toolkit (IGSTK) open source framework is used.

\subsection{High-level controller}

The HLC provides single robot controllers with the via points necessary to define the desired trajectory, solving any problems of redundancy in degrees of freedom and avoiding singularities. The HLC is also responsible for avoiding collisions with devices in the OR and patient, assuring patient mechanical safety. With this aim, envelopes of the patient and of the OR instruments are collected by the optical tracker and produced via generic models. The control is actuated by means of Petri nets that are utilized to solve for parallel workflows $[\mathbf{1 6}, \mathbf{1 7}]$.

\subsection{Human-computer interface}

As in the usual image-guided surgery, a visualization workstation is in charge of interfacing the software platform (for ROBOCAST, 3D Slicer 3.4 is used [18]) with the end user. Traditional methods are used for the registration of different images (CT, MR, etc. [19]) and for the determination of the target inside the brain (the surgeon makes a manual selection of the target on the medical images slices) while the planning phase is supported by the autonomous planner described hereafter (section 2.6). The graphical interface visualizes the path proposed, so that the surgeon can accept or criticize the planner's solution. Intra-operative replanning to compensate for brain shift will be carried out by intra-operative US imaging through a burr hole or temporal window.

The interface of ROBOCAST is endowed with a haptic device. A one-dimensional software constrained Omega (Force Dimension, Nyon, Switzerland) device [20]) is used to convey to the surgeon the resistance of the brain parenchyma to straight probe advancement. Amplification of linear motion and/or force will be provided. The force feedback information comes from the load cell sensing the force experienced by the tip of the probe during the advancement under LA control. Since a maximum speed limitation is also provided, this information is conveyed on screen (also with an audio warning) to distinguish the resistance of the haptic stick due to brain stiffness from the LA locking of maximum speed. 


\subsection{Autonomous planner}

The surgical planning requires accurate target identification and trajectory selection. The ROBOCAST project contribution to this task is oriented to the proposition of a possible optimal trajectory for reaching the target inside the brain with minimum damage to the patient.

The risk of damaging brain structures comes from crossing important anatomical regions (e.g. the ventricles or the pituitary gland), functional areas highlighted with fMRI analysis (e.g. vision areas, motor area, or Broca's area), blood vessels that become visible through magnetic resonance angiography (MRA) (or by computed tomography angiography (CTA) if present in the clinical protocol), fibre bundles that are tracked on DTI, and the target volume (such as the lesion, during biopsies or dialysis fluid sampling), all of which is outlined on T1 MR images. All the aforementioned structures constitute a subject-specific map which is considered for planning (as shown in Fig. 3) [21].
The anatomical risk map represents the importance of all the anatomical areas and therefore the risk for the patient if they are crossed with the surgical probe. Potential risks are haemorrhage, with consequent hemiplegia, functional loss, and other consequences. A table was built associating a risk value (from 0 indicating no risk, to 100 indicating extreme danger) with the anatomical structures. The list of structures and the corresponding atlas were derived by the Multimodal SPL Brain Atlas Data [22] where 160 anatomical structures were segmented on MR data $(1.5 \mathrm{~T}, 256 \times 256 \times 159$ with voxel size $0.93 \mathrm{~mm} \times 0.93 \mathrm{~mm} \times 1.5 \mathrm{~mm}$ ) acquired from a single normal subject. The anatomical risk map (specific to the patient) is derived, registering the risk atlas on the T1 MR images of the patient.

The vasculature risk map is automatically extracted from MRA images and expressed as a label map [23]; it is expressed as inversely proportional to the distance of the considered voxel to the nearest vessel.

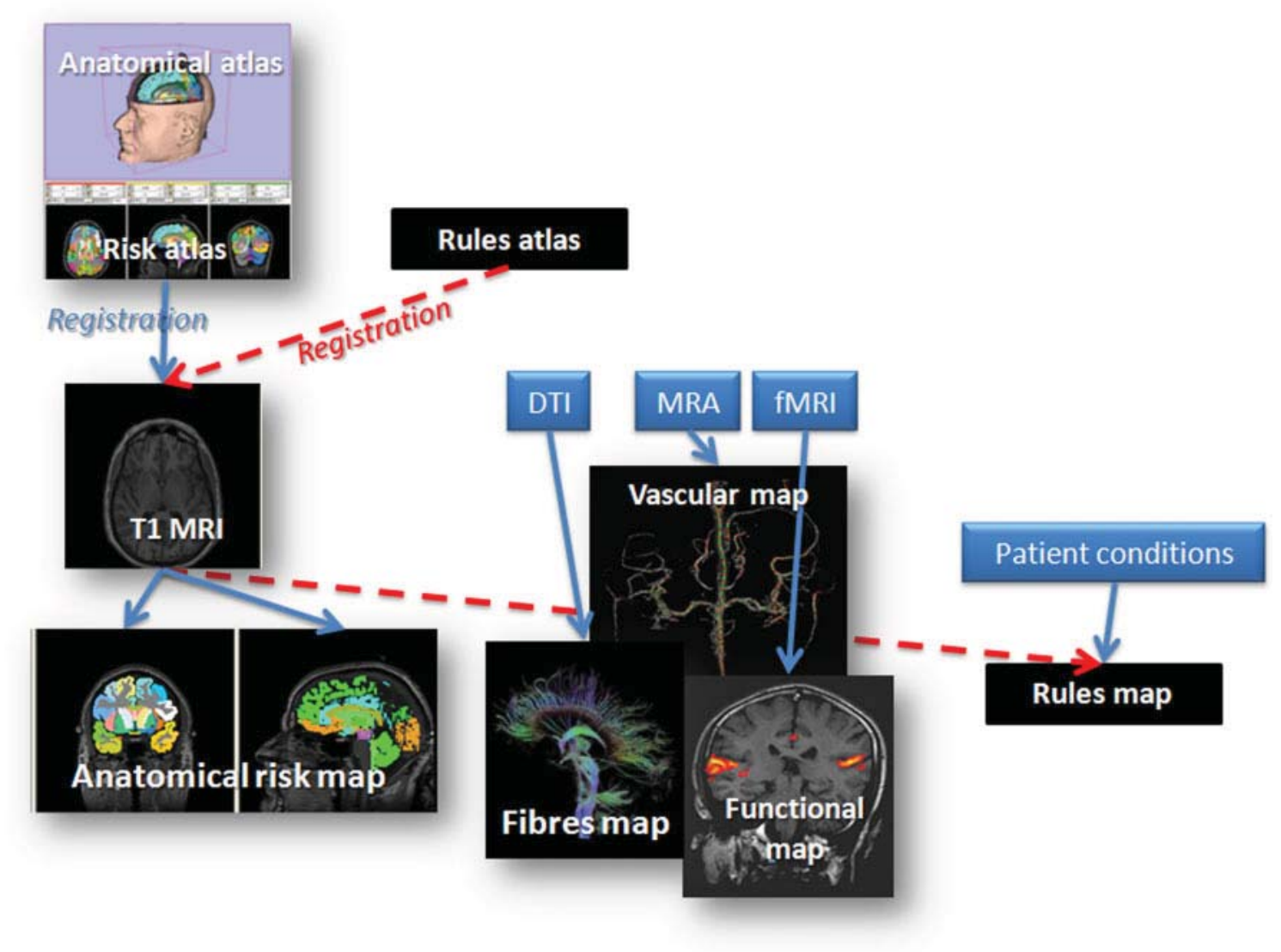

Fig. 3 Data flow to obtain the maps of the patient. The anatomical risk map is derived by the registration of the anatomical statistical atlas, with associated risk value, once it is registered on the T1 MR images of the patient. The fibre map is derived from DTI; the vascular map is derived from MRA (or CTA); and the functional map is computed using the fMRI data sets. The rule map is derived from the surgeon's knowledge of the patient conditions and, compulsorily, from his or her knowledge (dashed lines) stored in the database 
The functional map consists of functional areas that are extracted from the fMRI data of the patient, and is expressed as a label map. An importance value is associated with each function.

The fibre map shows fibres that are automatically extracted from the DTI data of the patient, and is expressed as a label map. An importance value is associated with each kind of fibre. Also, the fibre bundles risk map is expressed as inversely proportional to the distance of the considered voxel to the nearest fibres bundle.

In the target area map, the target volume is manually segmented by the user on T1 MR images, and a user-dependent risk value is associated with that volume.

The target point is also manually chosen and it is represented by a 3D point in the T1 MR space. The permitted entering regions on the skull are manually selected by the surgeon on the patient-specific head surface (outlined on the T1 MR images). This represents a heuristic input allowing the searching space to be reduced and sparing computation time. The voxels of these regions represent hard constraints for the automatic trajectory computation.

The cost function (CF) to be minimized depends on the five risk factors both linearly and non-linearly (through a threshold value) according to

$$
\begin{aligned}
\mathrm{CF}\left(N, x_{\mathrm{e}}, y_{\mathrm{e}}, z_{\mathrm{e}}, x_{\mathrm{t}}, y_{\mathrm{t}}, z_{\mathrm{t}}\right)= & \lambda_{1} \sum_{i=1}^{N} R_{1}(i)+\lambda_{2} \sum_{i=1}^{N} R_{2}(i) \\
& +\lambda_{3} \sum_{i=1}^{N} R_{3}(i)+\lambda_{4} \sum_{i=1}^{N} R_{4}(i) \\
& +\lambda_{5} \sum_{i=1}^{N} R_{5}(i) \\
& +\lambda_{6} \sum_{i=1}^{N}\left[R_{1}(i)>\mathrm{th}_{1}\right] \\
& +\lambda_{7} \sum_{i=1}^{N}\left[R_{2}(i)>\mathrm{th}_{2}\right] \\
& +\lambda_{8} \sum_{i=1}^{N}\left[R_{3}(i)>\mathrm{th}_{3}\right] \\
& +\lambda_{9} \sum_{i=1}^{N}\left[R_{4}(i)>\mathrm{th}_{4}\right] \\
& +\lambda_{10} \sum_{i=1}^{N}\left[R_{5}(i)>\mathrm{th}_{5}\right]
\end{aligned}
$$

where $R_{j}$ indicates the $j$ th risk ( $j=1$, vascular; $j=2$, fibre; $j=3$, anatomical; $j=4$, functional; $j=5$, target volume) value of the voxel $i(x, y, z)$ crossed by the planned trajectory. Each time that the risk associated with a voxel on the path overcomes the threshold $\mathrm{th}_{j}, \lambda_{j}$ is added to the summation (those values are representative of the importance of the represented structure). These thresholds account for very critical areas not to be crossed for long paths. $N$ is the number of voxels belonging to the trajectory starting from the entry point $\left(x_{\mathrm{e}}, y_{\mathrm{e}}, z_{\mathrm{e}}\right)$ and ending at the target point $\left(x_{\mathrm{t}}, y_{\mathrm{t}}, z_{\mathrm{t}}\right)$; thus a minimum-length criterion is implicit. An exhaustive search bounded by a heuristic input was performed.

In order to code the surgeon's knowledge and previous experience, a propositional atlas was developed. It allows the user to add propositions to all the labelled area (anatomical and target area, functional, fibres, and vessel if present). Fuzzy risk is coded as pseudo-natural language sentences easily understandable by the surgeon that can be created because of an intuitive interface. This interface includes preconditions, conditions, attributes, and actions. Currently only one action, namely $<$ avoid to cross $>$, is defined and the attributes are only allowed to $b e<$ never $>$ or $<$ as long as possible $>$. This choice guarantees that no conflicts can exist; only redundancy can be derived. In fact, a rule containing $<$ avoid to cross always $>$ will dominate any other $<$ avoid to cross as long as possible $>$. The latter, if referring to the same voxel, will be just redundant but will not conflict. Therefore a conflict arbitration is not necessary, and at the moment a redundancy pruning has not yet been implemented. The atlas is mapped on the patient space, together with the anatomical atlas. The rules are in fact associated with labelled voxels.

An automatic planning module was added to a 3D Slicer software platform. The user selects the maps that have to be considered during the path computation, depending on the image data sets available, the considered pathology, and whether the user wants to use the stored knowledge database. On the T1 MR images, the user can define the target point and 'NO GO' regions (free-hand drawing on the 2D image views, lines, or circles) that will therefore be labelled as unsafe and discarded during the trajectory computation. The surgeon can define the allowed entry regions, selecting their contours on the head surface. Starting from all the possible entry points on the head surface (exhaustive search), the algorithm computes all the possible trajectories 
towards the selected target point. For each crossed voxel, the risk value is computed using equation (1), and two checks are performed: whether it had been labelled as a 'NO GO' region or whether there are one or more propositions associated. All the paths not discarded are ordered with increasing risk values and, for equal risk values, with increasing length. All the possible paths are presented in the visualization environments to the surgeon, together with information regarding the path risk, length, and possible warning messages (derived from the stored explanations of the propositions). The user could add new propositions to the knowledge database if unsatisfied with the solutions provided.

\subsection{Results (case study)}

An expert neurosurgeon assigned importance values to all the atlas structures, from 20 to 30 in the case of motor or sensory deficit to 100 in the case of haematoma or coma. Targets were arbitrarily chosen by manually selecting fiducial points on the $2 \mathrm{D} \mathrm{T1}$
MR images of the patient and an entry region on the parietal lobe. The trajectory computation required a few seconds. Figure 4 shows an example of one of the computed trajectories; it passes through the right putamen (risk value, 20) in order to spare more critical areas and to minimize trajectory length.

\section{STATUS OF THE PROJECT}

The project started on 1 January 2008. During the first year the user requirements and the system specifications have been defined. In this framework the cooperation between technical and medical partners has been a great asset and two paradigmatic scenarios have been defined for the demonstrator. The main decisions were taken about hardware and software to be used, and the main components have already been procured by the partners. The partners agreed on the interfaces to be implemented and on the standards to be used (e.g. Tao-Corba for computer-computer and computer-robot communications). The first tests were performed on the

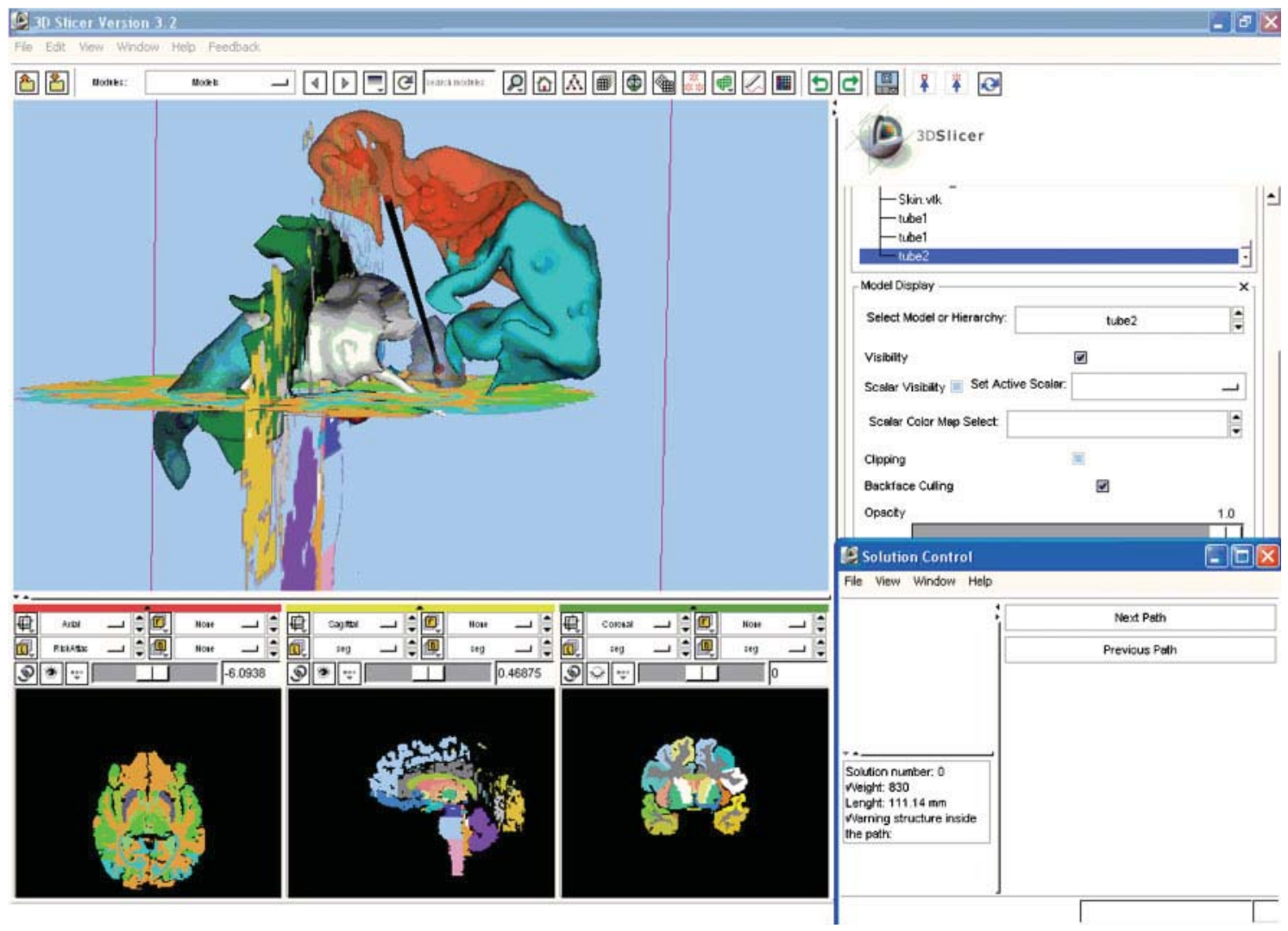

Fig. 4 The graphical user interface developed under 3D Slicer. At the top left, in the 3D visualization environment, the target point is visible (red spot), together with the anatomical structures (reconstructed as surface mesh) and the risk values (2D slice). At the bottom left are the 2D views of the risk and the anatomical maps. At the top right is the command panel, where the user has the possibility of loading maps 
shapes of the flexible probe for the analysis of the friction during the advancement [6]. A first smallscale version of a sample risk atlas has been built and the first tests have been made on the path planning. Computer-aided designs for the robots have been delivered to the control group in order to start simulations.

\section{CONCLUSIONS AND DISCUSSION}

The contribution that the ROBOCAST project wants to give to robotic surgery is the introduction of a modular and integrated ICT-based system for OR support. Up to now the user requirements, the architecture of the system, and its specifications have been produced. The strong points with respect to previous attempts, which appear viable at this stage of the project, are the following.

The system is supposed to be autonomous in the choice of the path to be followed, and its intelligent behaviour will allow incrementing of the knowledge of the system itself because of the new information given by the user as criticism. Use of atlases for trajectory planning in neurosurgery has already been proposed by some authors [7-9]. The approach here proposed steps forward in many aspects since more risk maps are used contemporaneously and taken into account with a non-linear cost function, which differently weights the several sources of information. Research is nowadays directed towards multimodality images visualization; the aim is to make it easily and immediately understandable by the surgeon [24], rather than to provide autonomous planning instruments. Currently, apart from the work by Chan et al. [25], who allowed the user to select the areas that have to be avoided, there is no system which automatically computes the best trajectory. Comparing this approach with other previous work [9], the present authors do not feel that an automatic assignment of tissue importance could be viable in such a complex and critical field as neurosurgery; thus the basic numerical risk information will be built in strict connection with neurosurgeons and neuroradiologists, rather than hypothesizing a system based on medical images contrast.

In parallel, upgradable fuzzy rules are considered, representative of the surgeon's experience, which, when updated on the field, can be applied while work is in progress without recompiling the program. The system guarantees consistency of the rule base by means of its technical construction, e.g. by underlying knowledge representation methods, since it cannot be left to the users to maintain the rule base and avoid conflicting entries. It may be necessary to provide offline quality maintenance of the rule base. This is made very simple because the knowledge is stored in a comprehensible way and easily upgraded using an intuitive user interface. Since neurosurgery is a dynamic field, there are provisions for easy rule withdrawal and modifications. Furthermore, as the annotated knowledge is possibly supplied by experts having non-congruent opinions, procedures to deal with conflicting advice have to be devised. An approach in agreement with the conservative medical domain would be either to resolve such situations by offline maintenance of the rule base before conflicts arise, or to present conflicting advice in terms of rich case descriptions, leaving it to the surgeon to judge the relevance of each case for the current situation. The path search is constrained to possible entry areas (heuristics) so as to reduce the search space and, consequently, to reduce the computational cost. Even if the case study showed promising results, further extensive tests are required, comparing the automatically computed trajectory with those selected by expert surgeons, e.g. in the case of challenging approaches such as the contralateral biopsy approach, where the vessels of the central sulcus have to be avoided. An improvement in the spatial resolution by a single order of magnitude would imply an increase in the representational effort by three orders of magnitude. More advanced concepts for spatial representations may be considered, e.g. octrees or $k$ d trees. All these characteristics, bringing artificial intelligence into play in the field of computer-assisted neurosurgery, represent a noticeable advancement with respect to previous work in the field.

The use of multiple-size robots, with redundant degrees of freedom, will allow probe positioning to be very precise while maintaining a wide range of motion and avoiding singularities; furthermore the new concept of biomimetic curvilinear probe that will be introduced will allow buckling problems to be avoided on turning. This part certainly poses more risk for the system; therefore a basic proof of concept is expected to be produced within the 3 years of the project. The current advancement of this part of the ROBOCAST project, after 1 year from its start, has led to the first studies on the materials and shapes for the biomimetic flexible probe [6].

The use of intra-operative redundant optical, EM, and US sensors will allow the reliability of the system to be increased and malfunctioning to be detected. The use of a Doppler sensor for sensing the presence 
of small vessels will increase patient safety during the operation. Cooperative control of the ROBOCAST system is achieved through a haptic interface that allows advancement of the surgical probe inside the brain, once the GP and the FP have autonomously reached their goal positions and orientations. The surgeon can actively manoeuvre the surgical instrument, achieving enhanced information regarding the resistance offered by brain tissues, filtering hand tremors, and stopping the advancement in the case of unexpected change in the sensed resistance or velocity which exceeds the maximum allowed. Haptic feedback will enrich the driving capability of the user, while keeping the security owing to speed, force, and direction constraints. Patient safety is considered; if the expected force is different from the actual force experienced during probe advancement, either the system is stopped if the difference is above a danger threshold, or a warning is sent to the operator.

A fruitful cooperation between surgeons, radiologists, and engineers is ongoing in the project in order to lead to a smoothly integrated, easy-to-use system for the operating room, avoiding the technological overcome of end user requirements.

The next 2 years will show whether the chosen approach is the actual answer to the current needs of MIS. Validation is expected on a gelatin phantom and, eventually, on a cadaver. It must be noted that the concept of risk maps, gathering together numerical as well as fuzzy information, and of the flexible probe, could easily be reused, the former not only in other medical applications involving different organs but also in very different fields, e.g. the exploration of dangerous environments. The incremental knowledge could, in fact, be used to manage risk after an accident has occurred. Each subsystem part of the ROBOCAST system could be reusable and also easily adapted and scaled to different applications which are not in the immediate scenarios of the project: the autonomous planner can be reused in all brain stimulation applications; with the stereotactic approach, the LA added to the FP (seventh degree of freedom) could be used as described in reference [26]; also, the sensor manager can incorporate other localization systems or imaging devices.

The system's cost-effectiveness will be considered in the exploitation phase, which will occur after the project is finished from a research and development viewpoint. The current system could be downgraded since the OR requirements are nevertheless supposed to be met.

\section{ACKNOWLEDGEMENTS}

The authors acknowledge the financial support of the EU Directorate General Information Society and Media, E1: Interaction and Interfaces (ROBOCASTFP7-ICT-2007-215190). The authors wish to thank Dr P. Tirelli and N.A. Borghese of Milan University, for collaboration in the algorithm for autonomous path planning, and all the partners working in the ROBOCAST project. Eleven partners are carrying out the research across Europe and Israel. The websites can be reached through the page 'consortium' of the project website: www.robocast.eu.

The coordinator of the project is the Bioengineering Department, Politecnico di Milano University, Milan, Italy, which is also working on the HCI, planning intelligence, and sensor integration. In charge of the main medical work packages is the Department of Neurological and Vision Sciences, University of Verona, Verona, Italy. Working on haptic device and software is the SIRSLab, Siena Robotics and System Lab, University of Siena, Siena, Italy. The Department of Mechanical Engineering and the Institute of Biomedical Engineering, Imperial College London, UK, is working mainly on the hardware platform and biomimetic flexible probe. Visualization and image processing is mainly carried out by the Computer-Aided Surgery and Medical Image Processing Laboratory, the Hebrew University of Jerusalem, Israel, and the Lehrstuhl für Computeranwendungen in der Medizin, Institut für Informatik, Technische Universität München, Germany. High-level control is the responsibility of the Institute for Process Control and Robotics, Universität Karlsruhe, Germany. The Faculty of Mechanical Engineering, Technion, Israel Institute of Technology, Haifa, Israel, is working on the FP and LA. Prosurgics Ltd, High Wycombe, UK, and Mazor Surgical Technologies Ltd, Cesarea, Israel, are in charge of providing robots and commercial exploitation of the results of the ROBOCAST project. CF Consulting, Finanziamenti Unione Europea S.r.l., Milan, Italy, is in charge of administration issues.

\section{(c) Authors 2010}

\section{REFERENCES}

1 Kiaii, B., Boyd, W. D., Rayman, R., Dobrowsi, W. B., Ganapathy, S., Jablonsky, G., and Novick, R. J. Robot-assisted computer enhanced closed-chest coronary surgery: preliminary experience using harmonic scalpel and Zeus. Heart Surg. Forum, 2000, 3, 194-197.

2 Mohr, F. W., Falk, V., Diegeler, A., Walther, T., Gummert, J. F., Bucerius, J., and Autschbach, R. 
Computer-enhanced 'robotic' cardiac surgery: experience in 148 patients. J. Thoracic Cardiovascular Surg., 2001, 121, 842-885.

3 Benabid, A. L., Hoffman, D., Ashraff, A., Koudsie, A., and Le Bas, J. F. Robotic guidance in advanced imaging environments. In Advanced neurosurgical navigation (Eds E. Alexander III and R. J. Maciunas), 1999, pp. 571-583 (Thieme Medical Publishers, New York).

4 Glauser, D., Fankhauser, H., Epitaux, M., Hefti, J. L., and Jaccottet, A. Neurosurgical robot Minerva: first results and current developments. J. Image Guided Surg., 1995, 1, 266-272.

5 Pandya, S., Motkoski, J. W., Serrano-Almeida, C., Greer, A. D., Latour, I., and Sutherland, G. R. Advancing neurosurgery with image-guided robotics. J Neurosurg, 2009, in press. DOI: 10.3171/ 2009.2.JNS081334.

6 Xia, T., Baird, C., Jallo, G., Hayes, K., Nakajima, N., Hata, N., and Kazanzides, P. An integrated system for planning, navigation and robotic assistance for skull base surgery. Int. J. Med. Robotics Comput. Assisted Surg., 2008, 4, 321-330.

7 Joskowicz, L. Advances in image-guided targeting for keyhole neurosurgery: a survey paper. In Touch Briefings reports, Future directions in surgery 2006, 2007, Vol. II, pp. 1-4 (Touch Briefings, London).

8 Vaillant, M., Davatzikos, C., Taylor, R. H., and Bryan, R. N. A path-planning algorithm for imageguided neurosurgery. In Proceedings of the First Joint Conference on Computer vision, virtual reality and robotics in medicine and medial robotics and computer-assisted surgery, Lecture Notes in Computer Science, Vol. 1205, Grenoble, France, 1922 March 1997, pp. 467-476 (Springer, Berlin).

9 Kaur, G., Tan, J., Alam, M., Chaudhary, V., Chen, D., Dong, M., Eltahawy, H., Fotouhi, F., Gammage, C., Gong, J., Grosky, W., Guthikonda, M., Hu, J., Jeyaraj, D., Jin, X., King, A., Landman, J., Lee, J., Li, Q. H., Lufei, H., Morse, M., Patel, J., Sethi, I., Shi, W., Yang, K., and Zhang, Z. CASMIL: a comprehensive software/toolkit for image-guided neurosurgeries. Int. J. Med. Robotics Comput. Assisted Surg., 2006, 2, 123-138.

10 Brunenberg, E. J. L., Vilanova, A., Visser-Vandewalle, V., Temel, Y., Ackermans, L., Platel, B., and ter Haar Romeny, B. M. Automatic trajectory planning for deep brain stimulation: a feasibility study. In Medical image computing and computerassisted intervention, Proceedings of the Tenth International Conference (MICCAI 2007), Lecture Notes in Computer Science, Vol.4791 (Eds N. Ayache, S. Ourselin, and A. Maeder). Brisbane, Australia, 29 October-2 November 2007, Part I, pp. 584-592 (Springer, Berlin).

11 Mazziotta, J., Toga, A., Evans, A., Fox, P., Lancaster, J., Zilles, K., Woods, R., Paus, T., Simpson, G., Pike, B., Holmes, C., Collins, L., Thompson, P., MacDonald, D., Iacoboni, M., Schormann, T., Amunts, K., Palomero-Gallagher, N., Geyer, S., Parsons, L., Narr, K., Kabani, N., Le Goualher, G.,
Boomsma, D., Cannon, T., Kawashima, R., and Mazoyer, B. A probabilistic atlas and reference system for the human brain: International Consortium for Brain Mapping (ICBM). Phil. Trans. $R$. Soc. Lond. B Biol. Sci., 2001, 356(1412), 1293-1322.

12 Eljamel, M. S. Validation of the PathFinder neurosurgical robot using a phantom. Int. J. Med. Robot., 2007, 3(4), 372-377.

13 Shoham, M., Lieberman, I. H., Benzel, E. C., Togawa, D., Zehavi, E., Zilberstein, B., Roffman, M., Bruskin, A., Fridlander, A., Joskowicz, L., Brink-Danan, S., and Knoller, N. Robotic assisted spinal surgery - from concept to clinical practice. Comp. Aided Surg., 2007, 12(2), pp. 105-115.

14 Frasson, L., Parittotokkapron, T., Schneider, A., Davies, B. L. D., Vincent, J. F. V., Degenaar, P., Huq, S. E., and Rodriguez y Baena, F. Biologically inspired microtexturing: Investigation into the surface topography of next-generation neurosurgical probes. In Proceedings of the 30th Annual International Conference of the IEEE Engineering in Medicine and Biology Society (EMBS 2008), 2008, pp. 5611-5614 (IEEE, New York).

15 Kulkarni, A. V., Guha, A., Lozano, A., and Bernstein, M. Incidence of silent hemorrhage and delayed deterioration after stereotactic brain biopsy. J. Neurosurg., 1998, 89, 31-35.

16 van der Aalst, W. M. P. The application of Petri nets to workflow management. J. Circuits, Systems Comput., 1998, 8(1), 21-66.

17 Russell, N., ter Hofstede, A. H. M., van der Aalst, W. M. P., and Mulyar, N. Workflow control-flow patterns: a revised view. BPM Center Report BPM06-22, 2006, p. 134, available from http://is.tm. tue.nl/staff/wvdaalst/BPMcenter/reports/2006/ BPM-06-22.pdf.

18 3D Slicer, 2009, available from http://www.slicer. org/.

19 Viola, P. A. and Wells III, W. M. Alignment by maximization of mutual information. Int. J. Comput. Vision, 1997, 24(2), 137-154.

20 Products overview, omega.x haptic devices, 2009, available from http://www.forcedimension.com/ products.

21 Vercauteren, T., Pennec, X., Perchant, A., and Ayache, N. Diffeomorphic demons: efficient nonparametric image registration. Neuroimage, 2008, 45(1, Suppl.), S61-S72.

22 Kikinis, R., Shenton, M., Iosifescu, D., McCarley, R., Saiviroonporn, P., Hokama, H., Robatino, A., Metcalf, D., Wible, C., Portas, C., Donnino, R., and Jolesz, F. A digital brain atlas for surgical planning, model-driven segmentation, and teaching. IEEE Trans. Visualization Comput. Graphics, 1996, 2(3), 232-241.

23 Frangi, A. F., Niessen, W. J., Vincken, K. L., and Viergever, M. A. Multiscale vessel enhancement filtering. In Medical image computing and computer-assisted intervention, Proceedings of the First International Conference (MICCAI '98), Lecture Notes in Computer Science, Vol. 1496 (Eds W. M. 
Wells, A. Colchester, and S. Delp). Cambridge, Massachusetts, USA, 11-13 October 1998, pp. 130-137 (Springer, Berlin).

24 Joshi, A., Scheinost, D., Vives, K. P., Spencer, D. D., Staib, L. H., and Papademetris, X. Novel interaction techniques for neurosurgical planning and stereotactic navigation. IEEE Trans. Visualization Comput. Graphics, 2008, 14(6), 1587-1594.

25 Chan, F., Kassim, I., Lo, C., Ho, C. L., Low, D., Ang, B. T., and Ng, I. Image-guided robotic neurosurgery - an in vitro and in vivo point accuracy evaluation experimental study. Surg. Neurol., 2009, 71, 640-648.

26 Joskowicz, L., Shamir, R., Freiman, M., Shoham, M., Zehavi, E., Umansky, F., and Shoshan, Y. Image-guided system with miniature robot for precise positioning and targeting in keyhole neurosurgery. Comput. Aided Surg., 2006, 11(4), 181-193. 